\title{
0492 THE COMPETENCY OF EMERGENCY MEDICAL OPERATION IN EMERGENCY MEDICAL PERSONNEL: ADVANCED LIFE SUPPORT UNIT
}

W Wijakkanalan* Correspondence: Sirindhorn College of Public Health, Khon Kaen, 90/1 Soi Anamai, Sricharn Road, Muang district, Khon Kaen 40000, Thailand

10.1136/ip.2010.029215.492

This research is descriptive research based on mixed methodology. The objective is designed to study the competency of emergency medical operation in emergency medical personnel: advanced life support unit in northeast of Thailand. The population used in the research includes the Advanced Life Support units from 19 northeastern provinces. The samples include Advanced Life Support units in 5 ambulance stations, chosen by multi-stage random sampling; Udonthani, Roi et, Nakhon Ratchasima, Surin, and Khon Kaen. The tools used in research, including Advanced Life Support unit record form, competency evaluation form of the Pre Hospital Registered Nurse (PHRN), Emergency Medical TechnicianIntermediate (EMT-I), Emergency Medical Technician-Basic (EMT-B) and First Responder (FR) in preparation, communication, scene size up, patient assessment, on scene care, care in transit, lifting and moving and consignment the patient. Data collection: documentary method, structured interview method, participant observation method and competency evaluation method is applied by using triangulation technique. Analysed by content analysis and typological analysis. Research found various range of outcome depended on the various competency of Advanced Life Support unit. Some PHRN gain improve, some can gain very good, EMT-I can gain fair to good, while the ambulance driver (EMT-B or FR) can gain improve to good.

Recommendation Emergency Medical Institute of Thailand should develop the competency that can not be scheduled of all level and review pre hospital care required to apply for the standard emergency medical operation protocol of Thailand. 